\title{
Andreev bound states for superconducting-ferromagnetic box
}

\author{
J. Cserti, ${ }^{1, \text { * J. Koltai, }}{ }^{2}$ and C. J. Lambert ${ }^{3, \text { 由 }}$ \\ ${ }^{1}$ Department of Physics of Complex Systems, Eötvös University \\ ${ }^{2}$ Department of Biological Physics, Eötvös University, \\ H-1117 Budapest, Pázmány Péter sétány 1/A, Hungary \\ ${ }^{3}$ Department of Physics, Lancaster University, Lancaster, LA1 4YB, UK
}

\begin{abstract}
Within the microscopic Bogoliubov-de Gennes (BdG) formalism an exact quantization condition for Andreev bound states of the ferromagnetic-superconducting hybrid systems of box geometry is derived and a semi-classical formula for the density of states is obtained. The semi-classical formula is shown to agree with the exact result, even when the exchange field $h$, is much larger than the superconductor order parameter, provided $h$ is small compared with the Fermi energy.

PACS numbers: 74.45.+c, 75.45.+j, 03.65.Sq
\end{abstract}

Mesoscopic hybrid systems formed from ferromagnets (F) in contact with superconductors (S) exhibit interesting transport properties resulting from the suppression of the electron-hole correlation in the ferromagnets 1.2.3.4.5.6.7.8. These transport phenomena are intimately related to the influence of an exchange field on the density of states (DOS) of clean ferromagnetic films in contact with superconductor, which has been investigated both experimentally $\underline{\underline{\underline{9}}}$ and theoretically ${ }^{10}$. In FS systems, Andreev bound states below the bulk superconducting gap are spin split by the exchange field of the ferromagnet. In a quasi-classical treatment of the diffusive regime it was shown that sub-gap features in the DOS of FS hybrids can be understood from the behavior of the length distribution of the classical trajectories existing in the ferromagnetic region, which depends purely on the geometry and the boundaries of the ferromagnetic region.

In this work we calculate the Andreev levels of an FS box consisting of a clean ferromagnetic region with a superconductor attached to one side, as shown in Fig. 1] This geometrical arrangement is a generalization of that investigated in Ref.10, where the size of the system along the FS interface was infinite. The discrete energy spectrum of the FS box system is obtained by solving the microscopic Bogoliubov-de Gennes (BdG) equation ${ }^{1.11}$. We derive an exact quantization condition without using the frequently applied Andreev's approximation. Our exact quantum description of the FS hybrid is an extension of the commonly used model developed by Blonder et al ${ }^{12}$ and by Saint-James and de Gennes 13 for normalsuperconducting systems. The mismatch in the effective masses and Fermi energies of the ferromagnet and the superconductor are taken into account in our calculations and the tunnel barrier at the interface is modeled using a Dirac delta potential. The treatment of the problem is based on an adaptation of a method developed in our previous work ${ }^{14}$ to the case of FS systems.

Starting from this exact quantization condition, we give a semi-classical expression for the sub-gap density of states (DOS) for exchange fields which are much less than the Fermi energy. The semi-classical DOS is expressed in terms of the classical return probability of

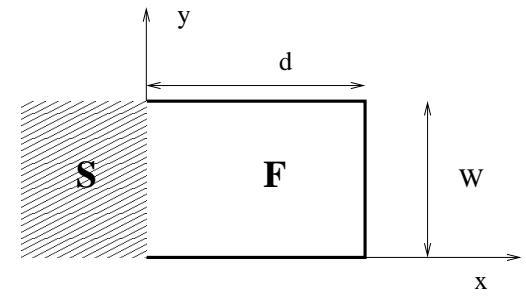

FIG. 1: A rectangular shape of ferromagnetic dot (F) in contact with a superconductor (S).

the electrons which depends only on the geometry of the $\mathrm{F}$ region and its boundaries. Besides the DOS, from our derivation an explicit expression for this return probability is also found. Based on the quasi-classical Green's function approach a similar expression has been derived for the DOS of ferromagnetic layers in contact with clean superconductor in Ref. 10. Our DOS expression for FS systems can be regarded as an extension of the Bohr-Sommerfeld formula developed for normalsuperconducting hybrids $\frac{15}{5}$. In this work we compare the DOS obtained from the exact quantum calculations with that found from our semi-classical formula. We show that a good agreement between the two calculations can be expected only for small enough exchange fields (much less than the Fermi energy).

The BdG equation for the FS systems can be written as

$$
\left(\begin{array}{cc}
H_{0}-\sigma h(\mathbf{r}) & \Delta \\
\Delta^{*} & -H_{0}-\sigma h(\mathbf{r})
\end{array}\right) \Psi_{\sigma}=E \Psi_{\sigma},
$$

where $H_{0}=\mathbf{p}^{2} / 2 m_{\mathrm{eff}}+V(\mathbf{r})-\mu$ is the single-particle Hamiltonian, $\mu=E_{\mathrm{F}}^{(F)}, E_{\mathrm{F}}^{(S)}$ are the Fermi energies, $m_{\mathrm{eff}}=m_{\mathrm{F}}, m_{\mathrm{S}}$ are the effective masses in the $\mathrm{F} / \mathrm{S}$ regions, $\Psi_{\sigma}$ is a two-component wave function, $E$ is the quasi-particle energy measured from the Fermi energy $E_{\mathrm{F}}^{(F)}$. Here $\sigma= \pm 1$ for spin up/down states and $+/-$ refer to the electron/hole like quasi-particle excitation. The tunnel barrier $V(\mathbf{r})$ at the FS interface and the exchange energy $h(\mathbf{r})$ are modeled in a usual way by $V(x, y)=U_{0} \delta(x)$ and $h(x, y)=h \Theta(x)$, where $\Theta$ is the 
unit step function. We also adopt the usual step-function mode1 1.12 for the pair potential and take $\Delta(\mathbf{r})=\Delta \Theta(x)$. It is easy to see that the Hamiltonian is separable and the ansatz for the wave functions in the $\mathrm{F}$ region can be written as

$$
\Psi_{m, \sigma}(x, y)=\left(\begin{array}{c}
a_{+} \sin \left[k_{m, \sigma}^{(+)}(x-d)\right] \\
a_{-} \sin \left[k_{m, \sigma}^{(-)}(x-d)\right]
\end{array}\right) \chi_{m}(y)
$$

while in the $\mathrm{S}$ region the wave functions have the form

$$
\Psi_{m, \sigma}(x, y)=\left(\begin{array}{l}
c_{+} \gamma_{+} e^{-i q_{m, \sigma}^{(+)} x}+c_{-} \gamma_{-} e^{i q_{m, \sigma}^{(-)} x} \\
c_{+} e^{-i q_{m, \sigma}^{(+)} x}+c_{-} e^{i q_{m, \sigma}^{(-)} x}
\end{array}\right) \chi_{m}(y)
$$

where

$$
\begin{aligned}
k_{m, \sigma}^{( \pm)} & =k_{\mathrm{F}}^{(\mathrm{F})} \sqrt{1 \pm \frac{E+\sigma h}{E_{\mathrm{F}}^{(\mathrm{F})}-\left(\frac{m \pi}{k_{\mathrm{F}}^{(\mathrm{F})} W}\right)^{2}}} \\
q_{m, \sigma}^{( \pm)} & =k_{\mathrm{F}}^{(\mathrm{S})} \sqrt{1 \pm i \frac{\sqrt{E^{2}-\Delta^{2}}}{E_{\mathrm{F}}^{(\mathrm{S})}}-\left(\frac{m \pi}{k_{\mathrm{F}}^{(\mathrm{S})} W}\right)^{2}} \\
\chi_{m}(y) & =\sqrt{2 / W} \sin (m \pi y / W) \\
\gamma_{ \pm} & =e^{ \pm i \arccos (E / \Delta)} .
\end{aligned}
$$

Here $m$ is a fixed integer and the Fermi wave numbers in the $\mathrm{F} / \mathrm{S}$ regions are given by $k_{\mathrm{F}}^{(\mathrm{F})}=\sqrt{2 m_{\mathrm{F}} E_{\mathrm{F}}^{(\mathrm{F})} / \hbar^{2}}$ and $k_{\mathrm{F}}^{(\mathrm{S})}=\sqrt{2 m_{\mathrm{S}} E_{\mathrm{F}}^{(\mathrm{S})} / \hbar^{2}}$. The wave functions satisfy the Dirichlet boundary conditions at the boundary of the $\mathrm{F}$ region except for the FS interface where the matching conditions ${ }^{14}$ should be applied. The four coefficients $a_{ \pm}, c_{ \pm}$in Eqs. (2a) and (2b) are determined from these matching conditions. One can find the following secular equation for the eigenvalues $E$ of the FS system for fixed mode index $m$ and spin state $\sigma$ :

$$
\operatorname{Im}\left\{\gamma_{+} \mathrm{D}_{\mathrm{m}, \sigma}^{(+)}(\mathrm{E}, \mathrm{h}) \mathrm{D}_{\mathrm{m}, \sigma}^{(-)}(\mathrm{E}, \mathrm{h})\right\}=0
$$

where $\operatorname{Im}\{$.$\} stands for the imaginary part and$

$$
\begin{aligned}
D_{m, \sigma}^{(+)}(E, h)= & \left(Z-i \frac{m_{\mathrm{F}}}{m_{\mathrm{S}}} q_{m, \sigma}^{(+)}\right) \sin k_{m, \sigma}^{(+)} d \\
& +k_{m, \sigma}^{(+)} \cos k_{m, \sigma}^{(+)} d, \\
D_{m, \sigma}^{(-)}(E, h)= & \left(Z+i \frac{m_{\mathrm{F}}}{m_{\mathrm{S}}} q_{m, \sigma}^{(-)}\right) \sin k_{m, \sigma}^{(-)} d \\
& +k_{m, \sigma}^{(-)} \cos k_{m, \sigma}^{(-)} d,
\end{aligned}
$$

and $Z=2 m_{\mathrm{F}} U_{0} / \hbar^{2}$ is the normalized barrier strength. The number of propagating modes for the electron/hole are the maximum of $m$ for which $k_{m, \sigma}^{( \pm)}$is a real number, i.e., $M_{\sigma}^{( \pm)}=\left[M \sqrt{1 \pm(E+\sigma h) / E_{F}^{(\mathrm{F})}}\right]$, where $M=$
$k_{\mathrm{F}}^{(\mathrm{F})} W / \pi$ and [.] stands for the integer part. For nonpropagating modes $k_{m, \sigma}^{( \pm)}$have to be replaced with their imaginary part and the functions sin and cos with the functions sinh and cosh, respectively. For fixed $m$ and $\sigma$ the solutions of Eq. (3a) for $E$ give the discrete sub-gap energy spectrum $(E<\Delta)$. These levels are numerically exact, i.e., no Andreev's approximation is used.

We now calculate density of states below the gap. In what follows, we assume that there is no mismatch and tunnel barrier at the FS interface $\left(m_{\mathrm{F}}=m_{\mathrm{S}}, E_{\mathrm{F}}^{(\mathrm{F})}=E_{\mathrm{F}}^{(\mathrm{S})}\right.$ and $Z=0$ ). For simplicity, we shall omit the superscript $\mathrm{F}$ and $\mathrm{S}$ in the wave numbers and the Fermi energies. In Andreev's approximation, i.e., for $|E+\sigma h| \ll E_{\mathrm{F}}$ we have $k_{m, \sigma} \approx q_{m, \sigma}$ and $D_{m, \sigma}^{(+)}(E, h) \approx k_{m, \sigma}^{(+)} e^{-i k_{m, \sigma}^{(+)} d}$. Therefore, the quantization condition (3a) can be simplified

$$
I_{m, \sigma}(E) \equiv \frac{\left(k_{m, \sigma}^{(+)}-k_{m, \sigma}^{(-)}\right) d-\arccos (E / \Delta)}{\pi}=n .
$$

The density of states for energies below the gap $(|E| \leq$ $\Delta$ ) is

$$
\varrho(E)=\sum_{\sigma= \pm 1} \sum_{n=-\infty}^{\infty} \sum_{m=1}^{M_{0}} \delta\left(E-E_{m n, \sigma}\right),
$$

where $E_{m n, \sigma}$ are the solutions of Eq. (4). Using (4) the DOS becomes

$$
\varrho(E)=\sum_{\substack{n=-\infty \\ \sigma= \pm 1}}^{\infty} \sum_{m=1}^{M_{0}}\left|\frac{d I_{m, \sigma}(E)}{d E}\right| \delta\left(I_{m, \sigma}(E)-n\right),
$$

where $M_{0}=M_{\sigma=+1}^{(-)}$, the number of propagating modes for spin-up-hole. Applying the Poisson summation formula 16 to the summation over $m$ and keeping only the non-oscillating term one finds

$$
\varrho(E)=\sum_{\substack{n=-\infty \\ \sigma= \pm 1}}^{\infty}\left|\frac{d I_{m, \sigma}(E)}{d E}\right|_{m=m^{*}} \frac{\Theta\left(M_{0}-m^{*}\right) \Theta\left(m^{*}-1\right)}{\left|\frac{\partial I_{m, \sigma}}{\partial m}\right|_{m=m^{*}}},
$$

where the $E$-dependent $m^{*}$ satisfies Eq. (4) for a given $n$ and $\sigma$. To simplify $I_{m, \sigma}$ we Taylor expand $k_{m, \sigma}^{( \pm)}$in terms of $E+\sigma h$ in first order (which is consistent with the Andreev's approximation) and find

$$
I_{m, \sigma}(E)=(E+\sigma h) \frac{2 d /\left(\pi \hbar v_{\mathrm{F}}\right)}{\sqrt{1-(m / M)^{2}}}-\frac{\arccos E / \Delta}{\pi},
$$

where $v_{\mathrm{F}}$ is the Fermi velocity. In our approximation, $M_{0} \approx M$. From (4) and using (8) we obtain

$$
\begin{aligned}
m^{*} & =M \sqrt{1-\left(\frac{2 d}{s_{n, \sigma}(E)}\right)^{2}}, \text { where } \\
s_{n, \sigma}(E) & =\frac{n \pi+\arccos (E / \Delta)}{(E+\sigma h) / \Delta} \xi_{0},
\end{aligned}
$$


and $\xi_{0}=\hbar v_{\mathrm{F}} / \Delta$ is the coherence length in the bulk superconductor.

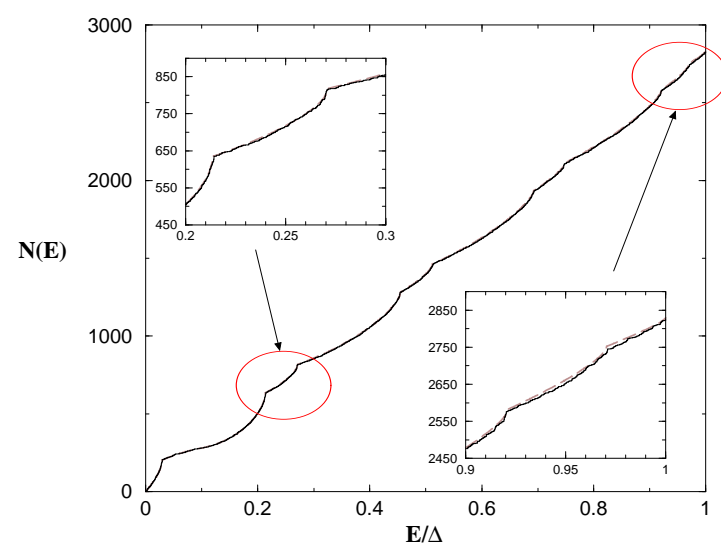

FIG. 2: Counting function $N(E)$ as a functions of $E / \Delta$ obtained from the exact (solid line) and the semi-classical calculation (dashed line) for $h / \Delta=0.1\left(h / E_{F}=0.0025\right)$. The other parameters are $M=217.7, d / W=0.7, \Delta / E_{\mathrm{F}}=0.025$. The insets show the enlarged part of the main frame.

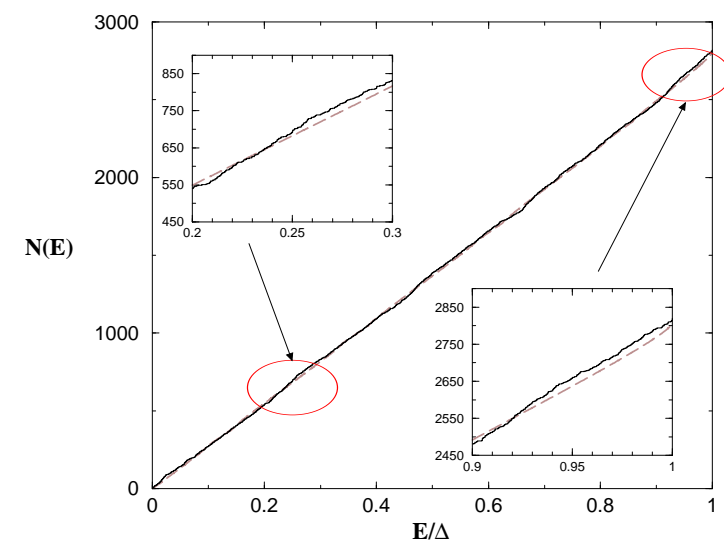

FIG. 3: Counting function $N(E)$ as a functions of $E / \Delta$ obtained from the exact (solid line) and the semi-classical calculation (dashed line) for $h / \Delta=10.0\left(h / E_{F}=0.25\right)$. The other parameters are the same as in Fig. 2 The insets show the enlarged part of the main frame.

Using (8) and performing the derivatives in (7) we find

$\varrho(E)=\sum_{\sigma= \pm 1} \frac{M}{|E+\sigma h|} \sum_{n=-\infty}^{\infty}\left[s_{n, \sigma}(E)+\xi_{c}(E)\right] P\left(s_{n, \sigma}(E)\right)$,

where

$$
P(s)=\frac{4 d^{2}}{s^{3} \sqrt{1-\left(\frac{2 d}{s}\right)^{2}}} \Theta(s-2 d),
$$

is a purely geometry-dependent function, $\xi_{c}(E)=$ $\xi_{0} / \sqrt{1-E^{2} / \Delta^{2}}$ and $s_{n, \sigma}(E)$ is given by Eq. (9). It can be shown that $P(s)$ is the classical probability that an electron entering the billiard at the FS interface returns to the interface after a path length $s$. The distribution $P(s)$ is normalized to one, i.e., $\int_{0}^{\infty} P(s) d s=1$. Note that the result for the DOS, given in Eq. (10) differs from that obtained in Ref. 10 by a factor multiplying $P(s)$ in the summation.

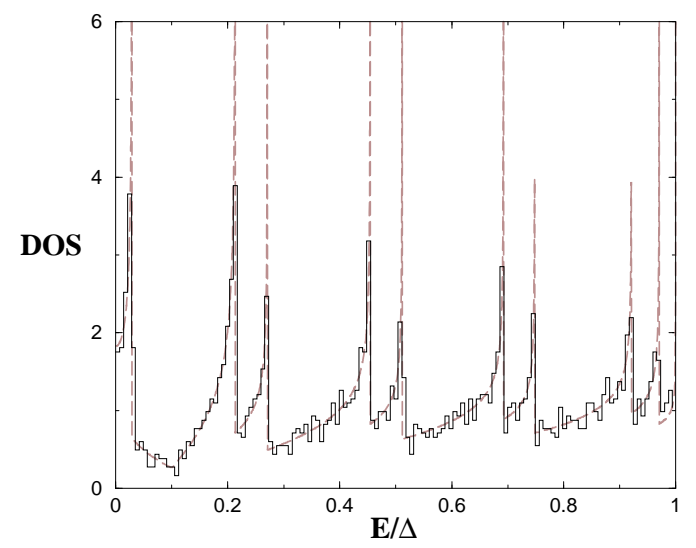

FIG. 4: The density of states $\varrho(E)$ as a functions of $E / \Delta$ (in units of $2 \varrho_{\mathrm{N}}$ ) obtained from the exact (solid line) and the semi-classical calculation (dashed line) for parameters given in Fig. 2

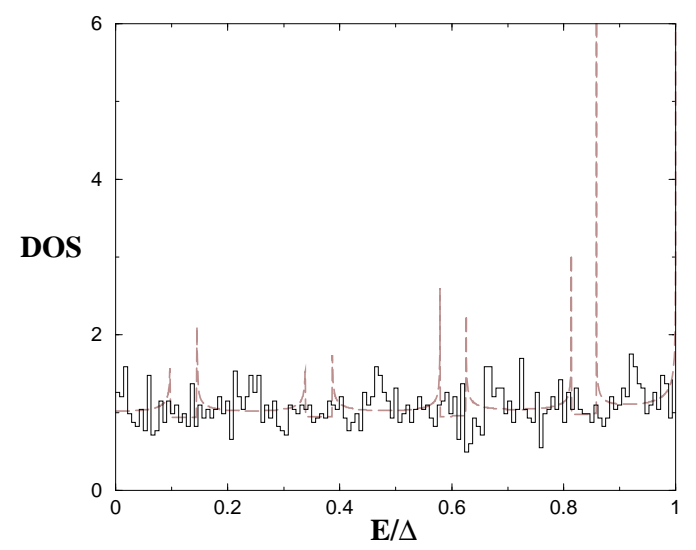

FIG. 5: The density of states $\varrho(E)$ as a functions of $E / \Delta$ (in units of $2 \varrho_{\mathrm{N}}$ ) obtained from the exact (solid line) and the semi-classical calculation (dashed line) for parameters given in Fig. [3]

To compare the exact DOS obtained from the quantization condition (3a) with that calculated from the semiclassical expression (10), we introduce the integrated 


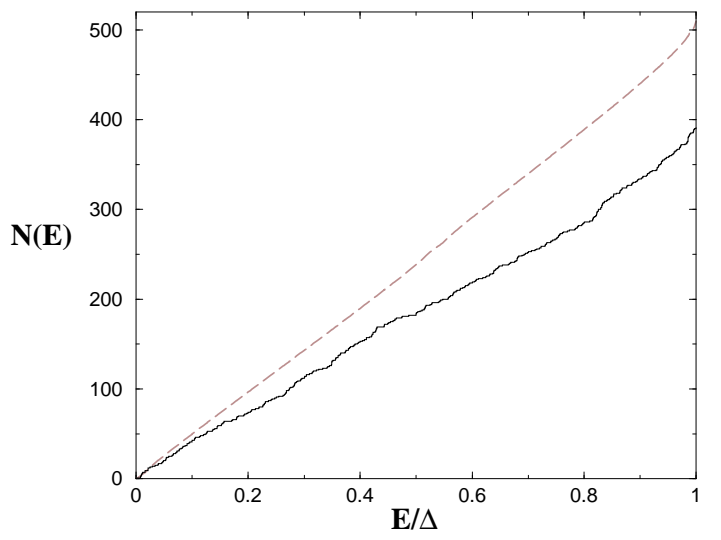

FIG. 6: Counting function $N(E)$ as a functions of $E / \Delta$ obtained from the exact (solid line) and the semi-classical calculation (dashed line) for $h / \Delta=40.0\left(h / E_{F}=1.0\right)$. The other parameters are the same as in Fig. 2

DOS: $N(E)=\int_{0}^{E} d E^{\prime} \varrho\left(E^{\prime}\right)$. From (10) we have

$$
\begin{aligned}
N(E) & =M \sum_{n=-\infty}^{+\infty}\left[F_{n}^{(a)}(E)+F_{n}^{(b)}(E)\right], \text { where } \\
F_{n}^{(a)}(E) & =F\left(s_{n,+1}(0)\right)-F\left(s_{n,+1}(E)\right), \\
F_{n}^{(b)}(E) & =\left\{\begin{array}{c}
1-F\left(s_{n,-1}(0)\right)-F\left(s_{n,-1}(E)\right), \text { if } E>h \\
-F\left(s_{n,-1}(0)\right)+F\left(s_{n,-1}(E)\right), \text { otherwise, }
\end{array}\right.
\end{aligned}
$$

and $F(s)=\int_{0}^{s} P\left(s^{\prime}\right) d s^{\prime}=\Theta(s-2 d) \sqrt{1-4 d^{2} / s^{2}}$ is the integrated length distribution. Note that $F(\infty)=1$ since $P(s)$ is normalized to 1 .

The small parameter used in reaching the semi- classical result is $h / E_{F}$. Figures 2 and 3 show a comparison between the semi-classical result Eq. (11) and the exact result Eq. (3), when $h / E_{F}=0.0025$ and 0.25 , respectively. For better resolution, in the insets enlarged parts of the main frames are shown. Figures 4 and 5 show the corresponding densities of states (in units of $2 \varrho_{\mathrm{N}}$, where $\varrho_{\mathrm{N}}=m_{\mathrm{F}} A /\left(\pi \hbar^{2}\right)$ is the DOS of the ferromagnetic region including spin up/down states and $A=W d$ is the area of this region) with singularities given by the semi-classical formula $s_{n, \sigma}\left(E_{\text {sing }}\right)=2 d$, where $\sigma= \pm 1$ and $n$ is such that $E_{\text {sing }}<\Delta$. These figures show that the semi-classical formula given by Eqs. (10)-(11) yield good agreement with the exact result, even for large values of $h / \Delta$, provided $h / E_{F}$ is small. Figure [6 shows a comparison with the exact result when the latter condition is violated. Clearly the agreement is poor in this limit.

In summary we have shown that a semi-classical treatment of the clean limit yields an expression for the DOS in terms of the classical return probability $P(s)$, which in turn is known analytically. This formula is analogous to the quasi-classical result of Ref. 10, where $P(s)$ is not known analytically and must be determined via a numerical simulation. We have also shown that the semiclassical formula agrees very well with the exact result for small exchange field compared with the Fermi energy.

We thank B. L. Györffy for helpful discussions. This work was supported in part by the European Community's Human Potential Programme under Contract No. HPRN-CT-2000-00144, Nanoscale Dynamics, the Hungarian-British Intergovernmental Agreement on Cooperation in Education, Culture, and Science and Technology, and the Hungarian Science Foundation OTKA TO34832.
* Electronic address: cserti@galahad.elte.hu

† Electronic address: c.lambert@lancaster.ac.uk

1 M. J. M. de Jong and C. W. J. Beenakker, Phys. Rev. Lett. 74, 1657 (1995).

2 I. Zutić and S. D. Sarma, Phys. Rev. B 60, R16322 (1999).

3 A. Kadigrobov, R. I. Shekhter, M. Jonson, and Z. G. Ivanov, Phys. Rev. B 60, 14593 (1999).

${ }^{4}$ I. Z̆utić and O. T. Walls, Phys. Rev. B 61, 1555 (2000).

${ }^{5}$ W. Belzig, A. Brataas, Yu. V. Nazarov, and G. E. W. Bauer, Phys. Rev. B 62, 9726 (2000).

${ }^{6}$ F. Taddei, S. Sanvito, and C. J. Lambert, Phys. Rev. B 63, 012404 (2000).

7 K. Kikuchi, H. Imamura, S. Takahashi, and S. Maekawa, Phys. Rev. B 65, 020508 (2001).

8 M. Krawiec, B. L. Györffy, and J. F. Annett, Phys. Rev. B 66, 172505 (2002).

9 T. Kontos, M. Aprili, J. Lesueur, and X. Grison, Phys. Rev. Lett. 86, 304 (2001).

10 M. Zareyan, W. Belzig, and Yu. V. Nazarov, Phys. Rev. Lett. 86, 308 (2001); M. Zareyan, W. Belzig, and Yu. V. Nazarov, Phys. Rev. B. 65, 184505 (2002).
11 P. G. de Gennes, Superconductivity of Metals and Alloys (Benjamin, New York, 1996).

12 G. E. Blonder, M. Tinkham, and T. M. Klapwijk, Phys. Rev. B 25, 4515 (1982).

13 P. G. de Gennes and D. Saint-James, Phys. Lett. 4, 151 (1963).

14 J. Cserti, A. Bodor, J. Koltai, and G. Vattay, Phys. Rev. B 66, 064528 (2002).

15 J. A. Melsen, P. W. Brouwer, K. M. Frahm, and C. W. J. Beenakker, Eur. Phys. Lett. 35, 7 (1996); A. Lodder and Yu. V. Nazarov, Phys. Rev. B 58, 5783 (1998); H. Schomerus and C. W. J. Beenakker, Phys. Rev. Lett. 82, 2951 (1999); W. Ihra, M. Leadbeater, J. L. Vega, and K. Richter, Eur. Phys. J. B 21, (2001).

16 M. Berry, in Chaos and Quantum Physics, Les Houches Summer School, edited by M.-J. Giannoni, A. Voros, and J. Zinn-Justin (Elsevier Science B. V., Amsterdam, The Netherlands, 1991); M. Brack and R. K. Bhaduri, in Semiclassical Physics, edited by D. Pines (Addison-Wesley Pub. Co., Inc., Amsterdam, The Netherlands, 1997). 